\title{
Arbeitsrechtspolitik ohne Tatsachengrundlage
}

\section{Eine Analyse der den Änderungen des Kündigungsschutzgesetzes zugrundeliegenden empirischen Annahmen ${ }^{1}$}

In ibrem Koalitionsvertrag vom I I.I I.200s hat sich die große Koalition auf eine "Weiterentwicklung des Kündigungsschutzgesetzes" (KSchG) geeinigt. Ziel dieser Gesetzesänderung soll sein, "mehr Beschäftigung zu ermöglichen ". Dieses Ziel soll dadurch erreicht werden, dass in neu abgeschlossenen Arbeitsverträgen eine Wartezeit von 24 Monaten (jetzt: 6 Monate) vereinbart werden kann. Erst nach dieser Wartezeit soll der Kündigungsschutz greifen. Dafür soll die Möglichkeit, Arbeitsverträge in den ersten zwei Jabren sachgrundlos zu befristen, gestrichen werden (nur für Existenzgründer bleibt diese Möglichkeit für 4 Jabre erhalten). Durch diese Änderungen soll der Kündigungsschutz einfacher und die Zabl arbeitsgerichtlicher Verfabren und das Prozessrisiko der Arbeitgeber gesenkt werden.

Dies ist nicht die erste und wohl auch nicht die letzte Änderung des KSchG. Ziel der bisherigen Änderungen war immer die Schaffung von Beschäftigung. Der vorliegende Beitrag untersucht, ob der unterstellte Zusammenhang zwischen Kündigungsschutz und Beschäftigung empirisch nachweisbar ist. Außerdem wird der Frage nachgegangen, ob der Gesetzgeber verpflichtet ist, bei Gesetzesänderungen empirische Forschung zur Kenntnis zu nehmen.

\section{Zusammenhang zwischen Arbeitsrecht und Arbeitsmarkt}

\section{a) Einstellungshemmnis Kündigungsschutz? Die aktuelle Diskussion}

Das KSchG wird verbreitet als eine Ursache für die hohe Arbeitslosigkeit angesehen. ${ }^{2}$ Es halte Betriebe davon ab, neue Beschäftigte einzustellen, obwohl dies von der anfallenden Arbeit her möglich wäre. ${ }^{3}$ Denn die Beendigung eines Arbeitsverhältnisses sei außerordentlich schwierig, das KSchG selbst für Fachleute nicht zu überblicken. ${ }^{4}$ Entschließt sich ein Betrieb dennoch zu einer Kündigung, erwarte ihn mit hoher Wahrscheinlichkeit eine teure gerichtliche Auseinandersetzung, deren Ergebnis nicht prognostizierbar sei.5 Aus Angst vor

I Beitrag für den Studienwettbewerb der Körberstiftung 2004 zum Thema: Mythos Markt? Die ökonomische, rechtliche und soziale Gestaltung der Arbeitswelt (2. Preis).

2 Für die juristische Diskussion: Rüthers, NJW 2002, I60I; Willemsen, NJW 2000, 2779; Hromadka, AuA 2002, 26I und ZfA 2002, 383; Bauer, NZA 2002, 529; Buchner, NZA 2002, 533; Neef, NZA 2000, 7; Busch, BB 2003, 470.

3 Für viele: Jerger, Wirtschaftsdienst 2003, 2 I s (216); Bauer, NZA 2002, 530; Hromadka, AuA 2002, 26I; Dr. Reinard Göhner, Bundesvereinigung deutscher Arbeitgeberverbände (BDA), auf einer Podiumsdiskussion des Hans-Böckler-Forums für Arbeits- und Sozialrecht am I 5.04.2005 in Berlin.

4 Jerger, Wirtschaftsdienst 2003, 216; Buchner, NZA 2002, 534.

5 Willemsen, NJW 2000, 2279 f.; Hromadka, Wirtschaftsdienst 2003, 223 (225). 
einem arbeitsgerichtlichen Verfahren würden die Betriebe zu teuren außergerichtlichen Abfindungsvergleichen gezwungen. Gerade betriebsbedingte Kündigungen, und hier vor allem der Nachweis des betrieblichen Erfordernisses und die Sozialauswahl, seien in der Praxis schwierig. ${ }^{6}$ Die mit einer rechtlichen Auseinandersetzung verbundenen hohen Kosten würden von den Betrieben antizipiert. Zu ihrer Vermeidung würde - vor allen Dingen in Kleinst- und Kleinbetrieben7 - auf Einstellungen verzichtet. Diese Darstellung des Zusammenhangs zwischen Arbeitsrecht und Arbeitsmarkt ist in der öffentlichen Diskussion fast unwidersprochen. Deutschland brauche eine neue Arbeitsverfassung, arbeitsplatzvernichtende Verkrustungen und Überregulierungen im deutschen Arbeitsmarkt müssten aufgebrochen werden, so die Position der BDA. ${ }^{8}$ Nicht nur das Handelsblatt ${ }^{9}$ und die Süddeutsche Zeitung ${ }^{1 \circ}$ berichteten im Frühjahr und Sommer 2003 regelmäßig über die Schicksale einzelner, vom Arbeitsrecht, speziell vom Kündigungsschutz gegeißelter Unternehmer. Auch im Wahlkampf 2005 waren solche Geschichten in den Medien häufig zu hören. Andere Gründe für die anhaltend hohe Arbeitslosigkeit wie die geringe Inlandsnachfrage und die schwache Konjunktur sind darüber fast vollständig in den Hintergrund geraten.

\section{b) Gesetzesänderungen und ibre Begründung}

Die soeben skizzierte Darstellung der angenommenen Wirkung des Kündigungsschutzes auf das Einstellungsverhalten der Arbeitgeber hat in der Vergangenheit schon mehrmals zu Gesetzesänderungen geführt. Im Rahmen des »Arbeitsrechtlichen Beschäftigungsförderungsgesetzes « vom 25.9.1996 ${ }^{\text {I I }}$ hatte der Gesetzgeber den Schwellenwert für die Anwendbarkeit des Kündigungsschutzgesetzes in $\ 23$ Abs. I Satz $2 \mathrm{KSchG}$ von 5 auf i० Beschäftigte heraufgesetzt und das Recht der betriebsbedingten Kündigung neu gestaltet. Die Sozialauswahl nach $\int$ I Abs. 3 KSchG wurde auf die Kriterien Lebensalter, Betriebszugehörigkeit und Unterhaltspflichten beschränkt. ${ }^{\mathrm{I} 2}$ Außerdem wurde im September 1996 mit dem neu eingefügten Absatz 4 des $\$ I KSchG die gerichtliche Überprüfbarkeit der betriebsbedingten Kündigung bei Vorliegen einer sogenannten »Namensliste in einem zwischen Betriebsrat und Arbeitgeber beschlossenen Interessenausgleich beschränkt und mit dem Absatz 5 die Beweislast für die Betriebsbedingtheit dieser Kündigungen umgedreht. Die durch die Bundestagswahl vom 27.9.1998 an die Regierungsmacht gelangte »rot-grüne« Regierungskoalition machte diese Regelungen sogleich durch das »Gesetz zu Korrekturen in der Sozialversicherung und zur Sicherung der Arbeitnehmer-

6 Berkowsky, DB I996, 778.

7 Vgl. Rede des Rechtsanwalts Moll auf dem 65. Deutschen Juristentag am 22.09.2004 in Bonn, zum Abdruck vorgesehen.

8 Http://www.bda-online.de/www/bdaonline.nsf/id/Brebes-Bessch_DE/\$file/Bremsklötze\% 2obeseitigen \% 20-\% 20Beschäftigung \% 20schaffen.pdf.

9 Serie: »Die Fesseln des Arbeitsmarktes: Wie das deutsche Arbeitsrecht neue Jobs verhindert« in I8 Folgen, Handelsblatt vom 31.0.3. bis 25.04 .2003 .

Io SZ vom I0.02.2003, S. 20: »Quasi handlungsunfähig « Unternehmen beklagen starres Arbeitsrecht/Betriebsräte verweisen auf innovative Lösungen.

I I BGBl. I S. I 476 .

I2 Gestrichen wurde durch das Arbeitsrechtliche BeschFG 1996 in $\$ I Abs. 3 S. I der unbestimmte Rechtsbegriff der »sozialen Gesichtspunkte«, die vom Arbeitgeber zu berücksichtigen seien. Ersetzt wurde dieser Begriff durch die Merkmale der Dauer der Betriebszugehörigkeit, das Lebensalter und die Unterhaltspflichten des Arbeitnehmers. Hinzu gefügt wurde im \ I Abs. 3 der Satz 2, wonach bestimmte Arbeitnehmer, deren Weiterbeschäftigung im berechtigten betrieblichen Interesse liegt, in die soziale Auswahl nicht einzubeziehen sind. 
rechte« vom I 9.I 2.I $198^{13}$ teilweise wieder rückgängig. Nur $\int$ I Abs. 4 Satz I und \ 23 Abs. I S. 3 KSchG (anteilig abgestufte Berücksichtigung von Teilzeitbeschäftigten in Kleinbetrieben; der Berechnungsschlüssel wurde allerdings $a b$ I.I.I999 zugunsten stärkerer Berücksichtigung von Teilzeitkräften etwas modifiziert) blieben im Wesentlichen erhalten. Der Schwellenwert wurde wieder auf fünf Beschäftigte abgesenkt, die Beschränkung der Sozialauswahl auf drei Kriterien und die sogenannte Namensliste abgeschafft. Dieselbe Regierung stellte ab OI.0I.2004 in nicht unwesentlichen Teilen der Rechtszustand des KSchG zwischen 1996 und 1998 wieder her: Die Kriterien der eingeschränkten Sozialauswahl wurden lediglich um das Kriterium der Behinderung ergänzt und der Schwellenwert nur für neu Eingestellte angehoben. Neu eingeführt wurde der in der Regierungserklärung des Bundeskanzlers Schröder vom I 5.03.2004 angekündigte »Abfindungsanspruch « bei Verzicht auf eine Klage gegen die Kündigung. Der Paragraf ist irreführend, denn ein »Abfindungsanspruch « im Wortsinne verbirgt sich hinter ihm nicht: Lediglich derjenige Gekündigte, der auf eine Klage verzichtet, hat einen Anspruch auf eine Abfindung, dem der Arbeitgeber diese Abfindung zuvor angeboten hat. Das war dem Arbeitgeber aber auch vor der Schaffung dieses Paragrafen möglich: eine Vereinbarung mit dem zu Kündigenden zu schließen, in der dieser auf sein Recht zur Klage verzichtet und der Arbeitgeber ihm dafür eine Abfindung zahlt.

In den Gesetzesbegründungen findet sich der oben skizzierte Wirkungszusammenhang zwischen Arbeitsmarkt und Kündigungsschutz wieder: Die KohlRegierung ging davon aus, dass »die Änderung des Kündigungsrechts und die Erleichterungen beim Abschluss befristeter Arbeitsverträge ... zu zusätzlicher Beschäftigung fübren "werden, da sich das komplizierte Kündigungsschutzrecht gerade in kleinen Betrieben auf die Einstellungsbereitschaft der Arbeitgeber auswirke. ${ }^{14}$ Dieses Einstellungshemmnis sollte besonders in Handwerksbetrieben bestehen, hier werde deshalb auch durch die Heraufsetzung des Schwellenwertes die Schaffung von Arbeitsplätzen prognostiziert: Deren Zahl wird sogar auf eine halbe Millionen geschätzt. ${ }^{I S}$ Außerdem wird die lange Dauer arbeitsrechtlicher Verfahren moniert ${ }^{16}$ - obwohl die offizielle Statistik der Arbeitsgerichte leicht zugänglich ist und das Gegenteil beweist. Empirische Forschung wurde nur für die Schaffung von Arbeitsplätzen durch die Erleichterung der Befristung, nicht jedoch für die Wirkung des Schwellenwertes auf das Einstellungsverhalten angeführt. Solche Forschungsergebnisse lagen zu diesem Zeitpunkt auch noch nicht vor.

Das Wiederherstellen des Zustandes von vor I 996 begründete die SchröderRegierung mit dem Argument, die Änderungen hätten die erhofften Wirkungen nicht erbracht. Im Übrigen sei die soziale Stabilität »eine wesentliche Voraussetzung für wirtschaftliches Wachstum und für die Schaffung neuer Arbeitsplätze ... Die 1996 vorgenommenen Einschnitte in Arbeitnebmerschutzrechte haben den sozialen Frieden und die soziale Partnerschaft als wichtige Rabmenbedingungen für Motivation und Leistung beeinträchtigt. Das Ziel, zusätzliche Arbeitsplätze zu schaffen, wurde nicht erreicht. Deshalb sollen die Einschränkungen des Kün-

I 3 BGBl. I S. 3843. Vgl. den Entwurf der Fraktionen SPD und BÜNDNIS 90/DIE GRÜNEN, BTDrs. I $4 / 45$ sowie die hierauf bezogenen Änderungsempfehlungen des Ausschusses für Arbeit und Sozialordnung, BT-Drs. I4/I5I, S. 22.

I 4 BT-Drs. I3/46I 2 (Entwurf eines arbeitsrechtlichen Gesetzes zur Förderung von Wachstum und Beschäftigung) vom I0.05.1996, S. I, 8.

is Ebd., S. 9 f.

I6 BT-Drs. I3/5107 (Entwurf eines arbeitsrechtlichen Gesetzes zur Förderung von Wachstum und Beschäftigung) vom 26.06.1996, S. 24. 
Gesetzesbegründung lag lediglich eine Untersuchung zu Schwellenwerten im Arbeitsrecht vor - mit dem Ergebnis, dass der Schwellenwert im Kündigungsschutz keine beschäftigungshemmende Wirkung habe. ${ }^{18}$

Diese Erkenntnis währte nicht lange: Die Wiederherstellung des Rechtszustandes zwischen 1996 und 1998 ab 2004 wurde damit begründet, Änderungen seien "dort notwendig, wo das geltende Kündigungsschutzrecht schwer handhabbar ist und sich starre Regelungen als Einstellungshemmnis erweisen. «19 Mittlerweile standen zwei weitere Untersuchungen zu Verfügung, aus denen sich ergab, dass die Anhebung des Schwellenwertes vermutlich nicht zur Schaffung neuer Arbeitsplätze führen würde. ${ }^{20} \mathrm{Im}$ Wahlkampf 2005 hatte die Union neue Vorschläge zur Reform des Kündigungsschutzes auf die politische Agenda gebracht: Zur Schaffung von Arbeitsplätzen sollte der Schwellenwert auf 20 erhöht und die Wartezeit - die Zeit, die ein Arbeitsverhältnis bestehen muss, bis der Kündigungsschutz greift - von 6 auf 36 Monate verlängert werden. Dies geschah vor dem Hintergrund von zwei weiteren Untersuchungen - übrigens eines Instituts welches nicht verdächtig ist, besonders arbeitnehmernah zu sein -, die belegen, dass die Veränderung des Schwellenwertes in der Vergangenheit nicht zur Schaffung von Arbeitsplätzen geführt hat. ${ }^{21}$ Die Große Koalition hat sich nun auf eine optionale Verlängerung der Wartezeit auf zwei Jahre geeinigt. ${ }^{22}$

\section{c) Ergebnisse der Arbeitsmarktforschung}

An der Wirklichkeitsdarstellung, die sich in Medien und Gesetzgebung wiederfindet, ist fast alles falsch: Erstens werden Kündigungen keinesfalls regelmäßig beklagt. Auch Abfindungen werden nur im Ausnahmefall gezahlt, so dass Kündigungen nur im Ausnahmefall teuer für die Unternehmen sind. Arbeitsgerichte sind die schnellsten der Republik. Konsequenterweise ist auch kein Einfluss des Kündigungsschutzes auf das Einstellungsverhalten der Betriebe nachweisbar.

Lag die Klagequote vor 25 Jahren - bei guter gesamtwirtschaftlicher Lage - bei ca. $8 \%,{ }^{23}$ wurde für Herbst 1999 bis Herbst 2000 durch Befragung von betroffenen Beschäftigten eine Klagequote von I I, I \% ermittelt. ${ }^{24}$ Für den Zeitraum I 998-2003 ergab sich durch eine Befragung von Personalverantwortlichen in den Betrieben eine Klagequote von I , ,3 \%, wobei kleinere Betriebe seltener beklagt wurden. ${ }^{25}$ Die Abweichungen ergeben sich möglicherweise daraus, dass die Befragung von Personalverantwortlichen nur bestehende Betriebe erfasste, während die Befragung der Betroffenen auch Gekündigte aus geschlossenen Betrieben erfasste. Es ist wahrscheinlich, dass Klagen bei Betriebsschließungen seltener

I7 BT-Drs.I4/45 vom I7.I I.I998 »Entwurf eines Gesetzes zu Korrekturen in der Sozialversicherung und zur Sicherung der Arbeitnehmerrechte

I 8 Friedrich/Hägele, Ökonomische Konsequenzen von Schwellenwerten im Arbeits- und Sozialrecht sowie die Auswirkungen dieser Regelungen - Kurzfassung des Endberichts, 1997.

I9 Entwurf eines Gesetzes zu Reformen am Arbeitsmarkt vom i 8.06.2003, BT-Drucks. I 5/I 204.

20 Wagner/Schnabel/Kölling, in: Ehrig/Kalmbach (Hrsg.): Weniger Arbeitslose - aber wie?, 200ı, S. I77; Pfarr/Bothfeld/Kaiser/Kimmich/Peuker/Ullmann, BB 2003, 2286.

2I Bauer/Bender/Bonin, Dismissal Protection and Worker Flows in Small Establishments, IZA discussion paper Nr.I105, April 2004; Verick, Threshold Effects of Dismissal Protection Legislation in Germany, IZA discussion paper Nr. 99I, Januar 2004.

22 Siehe Koalitionsvertrag zwischen CDU/CSU und SPD vom I I.I I.2005, S. 29 f.

23 Falke/Höland/Rohde/Zimmermann, Kündigungspraxis und Kündigungsschutz in der Bundesrepublik Deutschland, I98 I, Bd. I und II, S. 367.

24 Bielenski/Hartmann/Pfarr/Seifert, AuR 2003, 8I ff; weitere Ergebnisse der Studie: Bielenski/Hartmann/ Mauer/Seifert, WSI-Mitteilungen 2002, 442.

$25 \mathrm{Pfarr} /$ Bothfeld/Kaiser/Kimmich/Peuker/Ullmann, BB 2004, Io6. 
sind. Andererseits ist wahrscheinlich, dass letzterer Wert zu hoch ist. Denn die Personalverantwortlichen wurden in der Befragung nach den Klagen in den letzten 5 Jahren gefragt. Große Betriebe, die häufig mit Klagen konfrontiert sind, wurden gebeten, die Zahl zu schätzen, wenn die genaue Zahl nicht zur Hand war. Da negative Ereignisse häufiger über- als unterschätzt werden, ist es wahrscheinlich, dass der Wert bei größeren Betrieben häufig über dem realen Wert lag. ${ }^{26}$ Einen weiteren Einfluss könnte der Umstand gehabt haben, dass die gesamtwirtschaftliche Lage zum Befragungszeitpunkt jeweils variierte. Bekannt ist, dass die Wirtschaftslage einen Einfluss darauf hat, ob Kündigungen beklagt werden oder nicht. ${ }^{27}$ Das ist aus Sicht der Beschäftigten verständlich: Wer schnell eine neue Beschäftigung findet, wird die alte Kündigung seltener beklagen als jemand, der auf dem Arbeitsmarkt nur noch wenig Chancen hat.

Abfindungen werden nur in Is \% aller Beendigungen durch Arbeitgeberkündigung gezahlt, wobei Kleinstbetriebe mit bis zu ro Beschäftigten im Falle einer arbeitgeberseitigen Kündigung fast nie (nur in 4 \% der Fälle) Abfindungen zahlen. Auch bei kurzen Betriebszugehörigkeiten werden selten Abfindungen gezahlt. Erst ab einer Betriebszugehörigkeit von über is Jahren erhält jeder zweite Gekündigte eine Abfindung. Ab ca. s Jahren Betriebszugehörigkeit liegen die Abfindungen im Durchschnitt bei einem halben Monatsgehalt pro Beschäftigungsjahr, bei kürzeren Betriebszugehörigkeiten darunter. ${ }^{28}$

Wenn gegen eine Kündigung geklagt wird, ist auch das Verzugslohnrisiko nicht hoch: Zwei Drittel aller Beendigungsstreitigkeiten vor dem Arbeitsgericht sind nach spätestens 3 Monaten beendet. Nur $2 \%$ dauern länger als I Jahr. In Berufung gehen nur ca. $4 \%$ aller Verfahren. ${ }^{29}$

Die Ausgangsannahme - Kündigungen seien in der Regel mit hohen Kosten für die Unternehmen verbunden - ist demnach falsch. Das Gegenteil ist der Fall: Hohe Kosten bei einer arbeitgeberseitigen Beendigung von Arbeitsverhältnissen sind die Ausnahme, nicht die Regel. Deshalb verwundert es auch nicht, dass auch die abgeleitete Folge - das Kündigungsschutzgesetz verhindere durch seine hohen Kosten Einstellungen - nicht stimmt: Internationale Studien haben gezeigt, dass eine eindeutige negative Wirkung der Rigidität von Kündigungsschutzregeln auf das Beschäftigungsniveau im internationalen statistischen Vergleich nicht nachweisbar ist. Zu diesem Ergebnis kommen Truger und Hein nach der Auswertung von sieben internationalen Studien, die seit I 997 veröffentlicht wurden und die die Wirkungen von institutionellen kündigungsschutzrechtlichen Regelungen auf das Beschäftigungsniveau untersucht haben. $3^{\circ}$ Auch eine Studie der OECD aus 1999 zu dieser Frage hat gezeigt, dass ein rigider Kündigungsschutz keinerlei Auswirkung auf das Beschäftigungsniveau, sondern nur auf die Struktur von Beschäftigung und Arbeitslosigkeit hat. ${ }^{3 \mathrm{I}}$ Ebenso haben zwei multivariante Analysen auf zwei verschiedenen Datengrundlagen ergeben,

26 Eine ausführliche Diskussion aller vorhandenen Datenquellen findet sich bei Bielenski/Ullmann, Bundesarbeitsblatt 2005 , Heft I0, S. 4.

27 Frick /Schneider, Zunehmende Konfliktregulierung durch Arbeitsgerichte? Eine ökonomische Analyse der Häufigkeit von Kündigungsschutzprozessen, I999, S. 24.

$28 \mathrm{Vgl}$. Bothfeld/Ullmann, WSI-Mitteilungen 2004, S. 262; eine vertiefende Beschäftigung mit Einflüssen auf Abfindungszahlungen findet sich in Schneider/Ullmann, Abfindungszahlungen in der Praxis: Wer gewinnt, wer verliert?, zur Veröffentlichung vorgesehen in BB 4/2006.

29 Quelle: Statistik der Arbeitsgerichtsbarkeit, für die Arbeitsgerichte zusammengefasste Durchschnittswerte für die Jahre 1999 bis 2003 . Die Berufungsquote bezieht sich auf das Jahr $200 \mathrm{I}$.

30 Truger/Hein, Wirtschaftsdienst 2003, 509.

3 I OECD, Employment Outlook, 1999, S. 47 ff. 
kung hatte. ${ }^{2}$

Hierfür gibt es möglicherweise einen weiteren Grund - neben der Tatsache, dass arbeitgeberseitige Kündigungen in der Regel konfliktfrei und ohne Kosten durchzusetzen sind. Denn arbeitgeberseitige Kündigungen stellen nur etwa ein Drittel aller Beendigungen. Etwas häufiger kündigen die Beschäftigten selber. Durch Auslaufen einer Befristung endet ca. jedes 5. Arbeitsverhältnis. Etwa jedes Iо. wird einvernehmlich aufgehoben. 33 Wenn arbeitgeberseitige Kündigungen gar nicht den Hauptteil aller Beendigungen ausmachen, wird deutlich, dass ein Gesetz, welches diese Kündigungen an bestimmte Voraussetzungen knüpft, ebenfalls keinen entscheidenden Einfluss auf den Arbeitsmarkt haben wird.

Ein weiteres Argument gegen den Einfluss des Kündigungsschutzgesetzes auf die Einstellungspraxis der Unternehmen ist, dass das Arbeitsrecht in vielen Unternehmen gar nicht handlungsprägend ist: Das Arbeitsrecht wird entweder gar nicht berücksichtigt, es wird also aktiv dagegen verstoßen, oder die Unternehmen haben ihren eigenen Umgang mit ihm gefunden. Sie treffen ihre Entscheidungen flankiert vom Arbeitsrecht, aber nicht hervorgerufen durch das Arbeitsrecht. $\mathrm{Zu}$ diesem Ergebnis kommt ein Projekt der Hamburger Hochschule für Wirtschaft und Politik (HWP) zur Erforschung des Einflusses des Arbeitsrechts auf das Verhalten der Betriebe.34

Dieses Ergebnis ist in der Arbeitsmarktforschung nicht bestritten. ${ }^{5}$ Zwar gibt es immer wieder Befragungen, die meinen, das Gegenteil beweisen zu können. Zu der Erkenntnis, das KSchG verhindere Einstellungen, kommt beispielsweise eine Studie des Forsa-Instituts vom März 2003, die das Bundesministerium für Wirtschaft und Arbeit in Auftrag gegeben hatte. ${ }^{36}$ Danach sollen durch die Anhebung des Schwellenwertes rund 300.000 neue Stellen entstehen - in welchem Zeitraum, wird allerdings nicht gesagt. Gegen die Validität dieser Befragung gibt es erhebliche Einwände. So wird bei der Interpretation der Ergebnisse außer Acht gelassen, dass die Erhöhung der gesamtwirtschaftlichen Nachfrage die unbedingte und unumgängliche Voraussetzung für Neueinstellungen ist. In der Fragestellung wurde diese einfach vorausgesetzt. 37 Die Forsa-Fragen richteten sich nicht auf eine empirisch nachprüfbare, konkrete personalpolitische Praxis, sondern lediglich auf Einstellungsabsichten. Zuverlässige Aussagen über das tatsächliche Verhalten von Kleinstbetrieben lassen sich auf Grundlage dieser Daten nicht treffen. Damit sind die Befunde von Forsa eher als das Ergebnis einer Meinungsumfrage zu bewerten, in der die (politische) Einstellung der Kleinstbetriebe zum Kündigungsschutzgesetz erhoben wurde.

32 Bauer/Bender/Bonin (Fn. 2I), die Autoren benutzten für ihre Studie einen hierfür zusammengestellten Datensatz aus der Beschäftigtenstatistik, der Arbeitgeber und Beschäftigte in Betrieben mit bis zu $3 \circ \mathrm{Be}-$ schäftigten umfasst; Verick (Fn. 2I), benutzte für seine Analyse das IAB-Betriebspanel; Friedrich/Hägele, (Fn. I 8); Wagner/Schnabel/Kölling (Fn. 20).

33 Daten aus dem IAB-Betriebspanel sowie der WSI-Beendigungsbefragung, Pfarr/Bothfeld/Bradtke/Kimmich/Schneider/Ullmann, RdA 2004, 193 ff.

34 Bradtke/Fischer/Hübner/Schramm/Zachert, WSI-Mitteilungen 2004, I38; Schramm/Zachert, Arbeitsrecht - Personalpolitik - Wirklichkeit, 2004 .

35 Vgl. Redebeitrag von Prof. Sadowski auf dem 65. Deutschen Juristentag, die Beiträge sind zum Abdruck vorgesehen.

36 Im Rahmen der Forsa-Studie wurden ı०o I Betriebe mit I-s Beschäftigten befragt. iwd - Informationsdienst des Instituts der deutschen Wirtschaft Köln Nr. I7 vom 25. April 2003.

37 In der Untersuchung wurde gefragt, ob die Betriebe bei einer entsprechenden konjunkturellen Lage neue Mitarbeiter einstellen würden, wenn durch eine Änderung des Kündigungsschutzgesetzes sichergestellt wäre, dass damit nicht sogleich der Kündigungsschutz für alle Beschäftigten gelten würde. 
Auch eine Studie des Instituts der deutschen Wirtschaft (IW) $3^{3}$ meint herausgefunden zu haben, dass der Kündigungsschutz neue Arbeitsplätze verhindert. Es handelt sich auch hierbei um eine reine Meinungsumfrage, die allerdings eine überaus skeptische Haltung der Arbeitgeber gegenüber dem Arbeitsrecht widerspiegelt, die auch durch Daten der WSI-Befragung zur betrieblichen Personalpolitik (2003) belegt wird. 39 Die IW-Studie fragt z.B. nach den Bedingungen für Neueinstellungen und stellt als Antwortalternativen lediglich Verminderungen des arbeitsrechtlichen Schutzes zur Auswahl, nicht aber die Verbesserung der ökonomischen Lage des Unternehmens. Außerdem führen selbst solche Meinungsumfragen nur beim BDA-nahen IW zu dem Ergebnis, das Arbeitsrecht verhindere in großer Zahl Einstellungen. Die gemeinsame Studie des Manager Magazins und Watt Deutschland zieht aus ihrer Befragung folgendes Fazit: »Nur eine Minderbeit der befragten Unternebmen hat mit dem Tarif- und Arbeitsrecht Probleme. $30 \%$ geben an, sie hätten Probleme mit den Regulierungen des Arbeitsmarktes. Selbst Firmen in nicht guter Lage geben dies nur zu 44\% an. $44^{\circ}$

\section{Einschätzungsprärogative des Gesetzgebers}

So unbestritten diese Erkenntnisse sind, so wenig finden sie Eingang in die öffentliche Diskussion, die Medien und vor allen Dingen in die Gesetzesbegründungen. An diesem Widerspruch knüpft die Frage an: Darf der Gesetzgeber den aktuellen Stand von Forschung und Wissenschaft in seinen Gesetzesbegründungen einfach ignorieren oder muss er ihn zur Kenntnis nehmen und zumindest begründen, wenn seine Gesetzesvorhaben sich nicht danach ausrichten?

Rechtlich gesehen hat der Gesetzgeber einen weiten Gestaltungsfreiraum. Die getroffene Maßnahme muss nicht die effizienteste sein..$^{\text {I }}$ Sie muss nur grundsätzlich zur (verfassungsrechtlich legitimierten) Zielerreichung geeignet sein und darf nicht willkürlich erscheinen. Ein Mittel ist bereits dann im verfassungsrechtlichen Sinne geeignet, wenn mit seiner Hilfe der gewünschte Erfolg gefördert werden kann, wobei die Möglichkeit der Zweckerreichung genügt. ${ }^{22}$ Auf dem Gebiet der Arbeitsmarkt-, Sozial- und Wirtschaftsordnung kommt dem Gesetzgeber ein besonders weitgehender Einschätzungs- und Prognosespielraum zu; es ist vornehmlich die Aufgabe des Gesetzgebers, auf der Grundlage seiner arbeitsmarkt-, sozial- und wirtschaftspolitischen Vorstellungen und Ziele und unter Beachtung der Sachgesetzlichkeiten des betreffenden Sachgebiets zu entscheiden, welche Maßnahmen er im Interesse des Gemeinwohls ergreifen will.43

Aber auch ein Entscheidungsspielraum hat - wie jeder Raum - seine Grenzen. Das Parlament kann von diesem Spielraum nur Gebrauch machen und sich in seinem Rahmen bewegen, wenn es die Rechtstatsachen des zu regulierenden Bereichs zur Kenntnis und in Bezug nimmt. So führt das Bundesverfassungsgericht in seiner erneuten Cannabisentscheidung aus, Ausgangspunkt der verfassungsrechtlichen Prüfung sei die vom Gesetzgeber vorgenommene »konkrete

38 Janssen, iw-trends $2 / 2004$.

39 Pfarr/Bothfeld/Kaiser/Kimmich/Peuker/Ullmann, BB 2003, 2622 ff.

40 "Perspektive Mittelstand. Die deutsche Wirtschaft im Umbruch«, Dezember 2003, S. I0.

4I Zum Strafrecht: BVerfG, NJW 2004, 3620.

42 BVerfG, NZA 2005, I53 (I 54).

43 Ebd. 

bisprodukten ausgehende Gesundheitsgefahr anerkanntermaßen geringer sei als der Gesetzgeber bei Erlass des Gesetzes angenommen hatte, führte deshalb nicht zu einer Aufhebung der Vorschrift, weil gleichwohl nicht unbeträchtliche Gefahren und Risiken verblieben, so dass die Gesamtkonzeption des Gesetzes auch weiterhin vor der Verfassung Bestand habe. Hinsichtlich der Frage, ob die Strafbewehrung ein geeignetes Mittel zur Eindämmung des Gebrauchs der Droge Cannabis sei, sei die kriminalpolitische Diskussion noch nicht abgeschlossen. An wissenschaftlich fundierten Erkenntnissen über die Richtigkeit des einen oder des anderen Wegs fehle es. ${ }^{45}$ Aus diesen Ausführungen lässt sich der Umkehrschluss ableiten, dass die Einschätzungsprärogative des Gesetzgebers da endet, wo wissenschaftlich fundierte Erkenntnisse über die (Un)wirksamkeit eines bestimmten Weges vorliegen.

Für die Auffassung, der Gesetzgeber müsse vorhandenes empirisches Datenmaterial zur Kenntnis nehmen, streiten vier Verfassungsprinzipien: das demokratische Prinzip, das freiheitliche Prinzip, das rechtsstaatliche Prinzip und der Grundsatz der Gleichberechtigung.

\section{a) Das demokratische Prinzip}

Im demokratischen Prozess liegt das Versprechen, durch die Beteiligung verschiedener Interessengruppen einen gerechten Ausgleich zwischen den vorhandenen und ggf. widerstreitenden Interessen $\mathrm{zu}$ finden. In einer indirekten Demokratie geschieht dies nur selten durch die direkte Beteiligung der betroffenen Gruppen, sondern durch die Beteiligung gewählter Volksvertreter und -vertreterinnen. Ebenso wie die Wählerinnen und Wähler können die Abgeordneten aber nur dann zu einem gerechten Ausgleich der widerstreitenden Interessen kommen, wenn sie wissen, worüber sie sprechen. Andernfalls ist eine sachnahe Meinungsbildung in Parlament und Bevölkerung nicht möglich.

\section{b) Die Freiheitsrechte}

Im Grundgesetz stehen die Freiheitsrechte an erster Stelle. Das hohe Gewicht der Freiheitsrechte beinhaltet das Versprechen, dass ein zwischen verschiedenen Gruppen zu findender Kompromiss die Freiheitsrechte möglichst wenig einschränkt. Gewährleistet wird dies durch die Bindung von Recht und Gesetz an die Freiheitsrechte des Grundgesetzes. Durch die Wesentlichkeitstheorie und das Zitiergebot des Art. I9 I Satz 2 GG soll gewährleistet werden, dass nicht leichtfertig in Grundrechtspositionen Einzelner eingegriffen wird. Denn im Gegensatz zu Weisungen oder Verwaltungsvorschriften ist die Verabschiedung eines Gesetzes an bestimmte Regeln gebunden, die einerseits Öffentlichkeit, andererseits die Beteilung aller gewählten Volksvertreter und -vertreterinnen gewährleisten.

Aus Demokratie- und Freiheitsprinzip folgt daher, dass hauptsächliches Lenkungsinstrument des Gesetzgebers das Gesetz ist. Entgegen der Auffassung der sog. Positivisten beinhaltet jedoch auch das Gesetz an sich das Versprechen der sachlichen und moralischen Gerechtigkeit. Dies soll dadurch gewährleistet werden, dass in die Entscheidungen der Exekutive alle relevanten Argumente Eingang finden. Argumente, die mit dem Regelungsgegenstand nichts zu tun haben, 
dürfen in einem demokratischen Staat nicht Grundlage einer auf einem Gesetz fußenden Entscheidung sein. ${ }^{6}$ Sachliche Gerechtigkeit kann jedoch nur hergestellt werden, wenn die entscheidungserheblichen Tatsachen und auch die voraussichtliche Betroffenheit der Bevölkerung durch eine Regelung bekannt sind. Dies ist ohne eine Ermittlung der entscheidenden Rechtstatsachen nicht möglich. Für die Exekutive ist dies unbestritten - selbst dann, wenn ein Ermessensspielraum existiert.

\section{c) Das rechtsstaatliche Prinzip}

Das rechtsstaatliche Prinzip beinhaltet unter anderem das Prinzip der Gewaltenteilung, der allgemeinen Gleichbehandlung, des Vorranges der Verfassung und der Gesetze, die Gewährleistung von effektivem Rechtsschutz und das Recht auf den gesetzlichen Richter. Das Rechtsstaatsprinzip verspricht einerseits die Einhaltung einer bestimmten Form, die in Rechtssicherheit durch Rechtskraft resultiert. Andererseits wird durch das rechtsstaatliche Prinzip auch materieller Inhalt, beispielsweise die Verhältnismäßigkeit einer Entscheidung, versprochen. Der materielle Anspruch kann das Prinzip der Rechtssicherheit allerdings nur in seltenen Fällen durchbrechen: Im Strafrecht ist dies der Fall, wenn neue Beweismittel, die neue Tatsachen beweisen, aufgetaucht sind oder wenn sich später herausgestellt hat, dass das Urteil auf falschen Tatsachen fußt. Die Rechtskraft eines Urteils des Bundesverfassungsgerichts kann durch erneute Vorlage einer Rechtsfrage nur aufgehoben werden, wenn tatsächliche oder rechtliche Veränderungen eingetreten sind, die die Grundlage der früheren Entscheidung berühren und deren Überprüfung nahe legen.47 Die Rechtsanwender und -anwenderinnen müssen danach alle relevanten Tatsachen zur Kenntnis nehmen.

\section{d) Der Gleichbehandlungsgrundsatz}

Viertens erfordert es der Gleichheitssatz, dass »Gleiches gleich und Ungleiches ungleich« behandelt wird. Die für eine Gruppenbildung notwendigen Kriterien müssen erkennbar und mit dem Grundgesetz vereinbar sein. Um jedoch festzustellen, wer gleich und wer ungleich ist, und wer durch eine Regelung wie betroffen ist, muss wiederum die Realität zur Kenntnis genommen werden. Dieses Prinzip bindet den Gesetzgeber unmittelbar. Stärker als die zuvor genannten Verfassungsprinzipien wird hierdurch die Einschätzungsprärogative des Gesetzgebers eingeschränkt. Seinem Gestaltungsspielraum sind hier umso engere Grenzen gesetzt, je stärker sich die Ungleichbehandlung von Personen oder Sachverhalten auf die Ausübung grundrechtlich geschützter Freiheiten nachteilig auswirken kann..$^{8}$ Ist dies der Fall, prüft das Bundesverfassungsgericht im Einzelnen nach, ob für die vorgesehene Differenzierung Gründe von solcher Art und solchem Gewicht bestehen, dass sie die ungleiche Rechtsfolge rechtfertigen können. 


\section{a) Das Problem der Erforschung der Wabrheit}

Einziges Argument dafür, dass sich der Gesetzgeber über die Ergebnisse von Wissenschaft und Forschung hinwegsetzen dürfen soll, ist der Umstand, dass eine zu enge Bindung der Politik an die Wissenschaft die Handlungsfähigkeit der Politik gefährden könnte. Dann drohe eine »Diktatur der Wissenschaft«.

Denn die Realität lässt sich häufig schwer ermitteln, zumal wenn es um so subjektive Dinge wie Einstellungen geht. Allerdings gibt es einfach zu ermittelnde Tatsachen: Wie viele Klagen gegen Kündigungen es in der Bundesrepublik gibt, ist leicht zu zählen. Eine andere, viel schwieriger zu beantwortende Frage ist beispielsweise, welchen Einfluss dieser Umstand auf das Verhalten der Personalverantwortlichen in den Unternehmen hat. Diejenigen Tatsachen, die im Strafrecht die »inneren Tatsachen « genannt werden, also der gesamte Bereich der Motivationsforschung, sind daher weit schwerer zu ermitteln als äußere, beobacht- und zählbare Umstände. Das Handeln der Betroffenen wird hier von unterschiedlichen Handlungsanforderungen bestimmt. Ein einziger Umstand lässt sich nur schwer als entscheidende Determinante für das Handeln isolieren. Manchmal ist die Rechtswirklichkeit, die reguliert werden soll, daher sehr unübersichtlich. Je schwerer ein Zusammenhang zu ermitteln ist, desto schwerer ist auch zu beurteilen, ob ein Forschungsergebnis valide ist. Wurden alle entscheidungserheblichen Determinanten berücksichtigt? Wurde die Befragungsgruppe sorgfältig ausgesucht, so dass die Ergebnisse repräsentativ sind? Allerdings gibt es auch hierfür Kriterien, die es einer kundigen Person ermöglichen, den Aussagegehalt und die Schwächen einer Studie zu ermitteln; Juristen und Juristinnen besitzen diese Kunde nicht qua Ausbildung.

Die Schwierigkeit, das Handeln bestimmter Akteure und den Aussagewert empirischer Sozialforschung zu erfassen, darf allerdings nicht dazu führen, dass Forschungsergebnisse vom Gesetzgeber überhaupt nicht zur Kenntnis genommen werden. Die Unmöglichkeit, die Folgen konkreter Rechtsgestaltung ganz exakt abzuschätzen, berechtigt nicht dazu, völlig auf rechtstatsächliche Fundierung zu verzichten und beliebig anekdotischer Evidenz zu folgen. Dies gilt umso mehr, je schwerer der Eingriff ist, der durch das gesetzgeberische Verhalten hervorgerufen wird. Da abhängig Beschäftigte - wie die Bezeichnung schon sagt - zur Existenzsicherung vom Verkauf ihrer Arbeitskraft abhängig sind, ist ein Eingriff in diese Grundlage, beispielsweise durch den Abbau des Schutzes vor ungerechtfertigten Kündigungen, als erheblich anzusehen. Rechtspolitische Diskussionen von entscheidender Bedeutung für die Betroffenen sollten daher auf der Grundlage möglichst objektiver und repräsentativer Erkenntnisse geführt werden.

\section{b) Einschränkung der Einschätzungsprärogative durch die Pflicht, einschlägige Forschung zur Kenntnis zu nebmen?}

Dies schränkt keinesfalls den Einschätzungsspielraum des Gesetzgebers erheblich ein: Dort, wo empirische Forschung nicht zur Verfügung steht, nach wissenschaftlichen Kriterien nicht valide ist oder verschiedene Studien zu erheblich divergierenden oder unklaren Ergebnissen kommen, ist es ureigenste Aufgabe der Politik zu entscheiden, ob es einer Regelung bedarf und wie diese ggf. auszusehen hat. Wie der Begriff schon deutlich macht, hat der Gesetzgeber einen weiten Einschätzungsspielraum. Der Einschätzungsspielraum gibt dem Gesetz- 
geber das Recht, das Gewicht verschiedener Interessen festzulegen. Vor allen Dingen aber darf der Gesetzgeber Werte festlegen, die seine Politik leiten. Es ist ihm nicht verwehrt, eine Regelung zu treffen, die der Allgemeinheit möglicherweise mehr Kosten auferlegt als eine andere, die dafür aber bestimmte Ziele besser verwirklicht. Hierzu gehört das Recht des Gesetzgebers, seine Bürger und Bürgerinnen zu sozialem Verhalten zu verpflichten. Der Einschätzungsspielraum beinhaltet jedoch die Pflicht, die für diese Einschätzung erheblichen Tatsachen und hier die einschlägige empirische Forschung zur Kenntnis zu nehmen, denn andernfalls kann eine Ergebnisabschätzung nicht erfolgen. Diese Verpflichtung des Gesetzgebers bedeutet keinesfalls, dass Gesetzgebung sich dem Diktat empirischer und ökonomischer Argumente aus der Forschung unterwerfen muss, sondern lediglich, dass diese zur Kenntnis genommen werden und in den Abwägungsprozess einfließen müssen. Ein Recht, auf ungesicherter oder gar falscher Tatsachengrundlage Entscheidungen zu treffen, gibt es für staatliche Institutionen nicht.

Zur Grenze der Einschätzungsprärogative sei der Einwand diskutiert, der Einigungsvertrag wäre niemals unterzeichnet worden, wäre die Wirkungsforschung gesetzlich vorgeschrieben gewesen. Selbstverständlich kann der Gesetzgeber gravierende Veränderungen wie die deutsche Einheit beschließen, die nicht so sehr aus ökonomischer, wohl aber aus politischer oder historischer Sicht sinnvoll oder gar zwangsläufig erscheinen. Allerdings wäre eine solchen Untersuchung, wäre sie denn durchgeführt worden, in der Öffentlichkeit breit diskutiert worden. Dann hätte die Politik gute - historische, politische - Argumente dafür anführen müssen, warum die Einheit trotz der durch sie entstehenden hohen Kosten durchgeführt werden soll. Ein solches Vorgehen hätte den Diskussionsund Entscheidungsprozess für die Bevölkerung transparenter gemacht und möglicherweise auch die Akzeptanz des Verhandlungsergebnisses erhöht. Rechtswirkungsforschung und ihre Berücksichtigung durch den Gesetzgeber schränkt die Einschätzungsprärogative des Gesetzgebers daher nicht ein. Sie führt nicht zu einem Zurückdrängen der Politik durch ein Diktat von Kommissionen und Sachverständigen. Sie zwingt ihn nur, Gesetzesvorhaben besser zu begründen. Dies ist unter anderem eine Versicherung für die Qualität des politischen Entscheidungsprozesses und eine Versicherung dagegen, ausschließlich behaupteten und unbelegten ökonomischen Zwängen zu folgen.

\section{c) Die aktuelle Diskussion um den Arbeitsmarkt: Es regiert das Diktat der Ökonomie}

Bei der aktuellen Debatte um den Arbeitsmarkt scheint es im Gegensatz zur Debatte um die deutsche Einheit, als hätten andere Argumente als das ökonomische kein Gewicht. Der Kündigungsschutz verhindere Einstellungen, die aus ökonomischer Sicht sinnvoll wären. Also muss er gelockert werden. Einwänden haftet das Prädikat »gemeinschaftsschädigend « oder »arbeitsplatzvernichtend « an. Das ist schon deshalb erstaunlich, weil nicht nur der Gesetzgeber unmittelbar, sondern auch die Parteien des Arbeitsvertrages über die mittelbare Drittwirkung der Grundrechte an die im Grundgesetz festgelegten Grundfreiheiten als nicht-ökonomische Werte gebunden sind: die Berufsfreiheit des Art. I 2 I GG, die Sozialbindung des Eigentums in Art. I4 II GG, vor allen Dingen die Koalitionsfreiheit des Art. 9 III GG.

Die Beschränktheit der Diskussion geht so weit, dass selbst bestimmte ökonomische Argumente für den Kündigungsschutz keinen Eingang in die öffentliche 
Diskussion finden: dass sich Beschäftigte, die sozial abgesichert sind und sich vor ungerechtfertigten Kündigungen sicher wähnen, stärker mit einem Unternehmen identifizieren, dass sie eher bereit sind, in sogenanntes betriebsspezifisches Humankapital zu investieren und in der Konsequenz produktiver arbeiten. 49

\section{Einfluss der verkürzten Diskussion auf die politische Kultur}

Das Außerachtlassen der für die Entscheidungsfindung erheblichen (fehlenden) Wirkungen des KSchG auf den Arbeitsmarkt ist nicht ohne Auswirkung auf die Glaubwürdigkeit der Politik. Deren sachliche Erklärungsnot führt dazu, dass immer häufiger statt Sachargumenten scheinbare Notwendigkeiten behauptet werden: so das Argument, die Gesellschaft müsste jetzt »Mut zu Reformen« haben. Warum die Gesellschaft Mut zu Reformen haben muss und warum bestimmte Einschnitte in das soziale Netz ertragen werden sollen, wird nicht gesagt. Dieser Diskussion ist jedes sachliche, d.h. die Wirklichkeit zur Kenntnis nehmende, Argument abhanden gekommen. Reformen werden um der Reformen Willen durchgeboxt. Neun Mal hat Bundeskanzler Schröder in den sieben Jahren seiner Amtszeit mit Rücktritt gedroht, sollte das Parlament seine Reformen nicht mittragen, vor allen Dingen zur Durchsetzung der Agenda 2010 und der Hartz-Gesetze - ein Verhalten, das dem demokratischen Prozess eher abträglich ist. Zu diesem Verlust einer fundierten Diskussionskultur passt die Veränderung des Gesetzgebungsprozesses, welche bei den Hartz- und Agenda 2010-Gesetzen erkennbar wurde: Während die öffentliche Diskussion in vollem Gange war, war ein begründeter Gesetzesentwurf nicht erhältlich. Dieser wurde erst kurz vor den entscheidenden Abstimmungen im Parlament erstellt bzw. der Öffentlichkeit zur Verfügung gestellt. Eine fachliche Diskussion am Entwurf wurde hierdurch genauso unterbunden wie die Einbindung der Fachgremien der Ministerien. Die Prüfung der Entwürfe nach Gender-Gesichtspunkten - nach der rot-grünen Koalitionsvereinbarung ein Muss für jedes Gesetzesvorhaben ist unterblieben. Möglicherweise ist so zwar weniger aufgefallen, dass die für das Gesetzesvorhaben vorgebrachten Argumente nicht stichhaltig waren. Diejenigen, denen der demokratische Prozess bzw. eine fundierte Diskussion und Kritik am Herzen liegt, mussten sich mit ihrer Kritik zurückhalten, weil diese ernsthaft erst geäußert werden kann, wenn es auch was zu Kritisieren gibt.

Die Befürchtung, die vermehrte Einbeziehung der Wissenschaft in die Politik würde zu einer »Sachverständigendiktatur « führen, ist weniger begründet als die Befürchtung der Selbstdelegitimation des Gesetzgebers durch ein nicht vermittelbares, weil nicht begründetes Verhalten. Schafft es die Politik nicht, zu einer Entscheidungsfindung zurückzukehren, in der verifizierbare Argumente eine Rolle spielen, wird die »Politikverdrossenheit «, die sich ausdrückt in fehlendem Interesse, an Willensbildungsprozessen vor Ort und durch Wahlen mitzuwirken, sich nicht umkehren. Politiker und Politikerinnen verlieren hierdurch mehr und mehr die Legitimation für ihr Handeln. 
Die aktuelle Debatte um den Arbeitsmarkt muss als empirie-resistent bezeichnet werden. Die gängigen Annahmen der Diskussion erweisen sich bei einer Überprüfung als falsch. Die Empirieresistenz spiegelt sich nicht nur in der medialen Diskussion, sondern auch in den Gesetzesbegründungen der Arbeitsmarktreformen der letzten Jahre wieder. Dabei ist der Gesetzgeber aus verschiedenen verfassungsrechtlichen Prinzipien dazu verpflichtet, die entscheidungserheblichen Tatsachen und damit die einschlägige Forschung zur Kenntnis zu nehmen. Zwar gibt es für ein Handeln gegen diese Verfassungsgebote keine rechtliche Sanktion. Möglicherweise war jedoch der Protest gegen die Arbeitsmarktreformen die zwangsläufige gesellschaftliche Reaktion auf die sachlich nicht fundierten politischen Debatten.

Arbeitnehmerschutz und vor allen Dingen der Kündigungsschutz wurden geschaffen, um das Machtgefälle zwischen abhängig Beschäftigten und Arbeitgebern auszugleichen. Wenn also der Abbau von Arbeitnehmerschutz ohne sachliche Rechtfertigung gefordert wird, dann ist der Vorwurf nicht unberechtigt, dies geschehe, um das Machtgefälle wieder herzustellen, d.h. Arbeit zu verbilligen. Hierzu passt auch, dass in jüngster Zeit dieses Machtgefälle zwar nicht mehrheitlich, aber wiederholt in Frage gestellt wird. Der Abbau von Arbeitnehmerrechten verbreitert allerdings nur vermeintlich den Handlungsspielraum der Arbeitgeber: Zwar führt dies möglicherweise zu einer Verbilligung von Arbeit. Langfristig wird der Abbau von sozialem Schutz im Arbeitsrecht der Allgemeinheit wahrscheinlich eher schaden: Das Kapital einer Wissensgesellschaft wie Deutschland sind ihre Bürgerinnen und Bürger. Der Gesellschaft als Ganzes hilft ein hohes Bildungsniveau, motivierte Beschäftigte sowie das Ankurbeln der Binnenkonjunktur. Ein hohes Bildungsniveau und eine hohe Motivation erreicht man nur durch soziale Absicherung. Dem Arbeitsmarkt sowie der demokratischen Kultur in diesem Land würde es daher gut tun, die Ergebnisse empirischer Wirkungsforschung zur Kenntnis zu nehmen. 